\title{
The conflict between treatment optimization and registration of radiopharmaceuticals with fixed activity posology in oncological nuclear medicine therapy
}

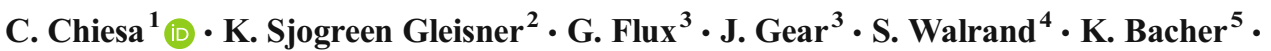 \\ U. Eberlein ${ }^{6}$ - E. P. Visser ${ }^{7}$ - N. Chouin ${ }^{8}$ M. Ljungberg ${ }^{2} \cdot$ M. Bardiès ${ }^{9} \cdot$ M. Lassmann $^{6}$ \\ L. Strigari ${ }^{10}$ M. W. Konijnenberg ${ }^{11}$
}

Received: 11 April 2017 / Accepted: 16 April 2017 /Published online: 24 May 2017

(C) Springer-Verlag Berlin Heidelberg 2017

\section{Formalization of the optimization principle in the new European Council Directive 2013/59}

The new European Council Directive 2013/59 (http://eur-lex. europa.eu/legal-content/EN/TXT/PDF/?uri=CELEX: 32013L0059\&from=EN), to be translated into national legislations before 6 February 2018, in article 56

C. Chiesa

carlo.chiesa@istitutotumori.mi.it

1 Nuclear Medicine Division, Foundation IRCCS Istituto Nazionale Tumori, Milan, Italy

2 Department of Medical Radiation Physics, Lund University, Lund, Sweden

3 Joint Department of Physics, Royal Marsden Hospital \& Institute of Cancer Research, Sutton, UK

4 Nuclear Medicine, Molecular Imaging, Radiotherapy and Oncology Unit (MIRO), IECR, Université Catholique de Louvain,

Brussels, Belgium

5 Department of Basic Medical Sciences, Division of Medical Physics, Ghent University, Ghent, Belgium

6 Department of Nuclear Medicine, University of Würzburg, Würzburg, Germany

7 Department of Radiology and Nuclear Medicine, Radboud University Medical Centre (RadboudUMC), Nijmegen, The Netherlands

8 LUNAM Université, Oniris, «AMaROC», Nantes, France

9 Centre de Recherches en Cancérologie de Toulouse, Université Paul Sabatier, UMR 1037 INSERM, Toulouse, France

10 Laboratory of Medical Physics and Expert Systems, National Cancer Institute Regina Elena, Rome, Italy

11 Department of Radiology and Nuclear Medicine Erasmus MC, Rotterdam, The Netherlands
(Optimisation) states: "For all medical exposure of patients for radiotherapeutic purposes, exposures of target volumes shall be individually planned and their delivery appropriately verified, taking into account that doses to non-target volumes and tissues shall be as low as reasonably achievable and consistent with the intended radiotherapeutic purpose of the exposure". No doubt this statement holds for nuclear medicine therapy, since in article 4 of the same, directive definition 81 states that "radiotherapeutic" means pertaining to radiotherapy, including nuclear medicine for therapeutic purposes. The directive thus asks for dosimetry, as is routinely implemented in radiotherapy, using external beam or brachytherapy sources. However, in nuclear medicine therapy, absorbed dose planning is rarely performed. One of the main reasons is the amount of work needed for internal dosimetry that includes multiple whole-body counts or scintigraphy and sometimes blood samples over some days after administration.

The "intended purpose" in all therapeutic exposures is treatment efficacy against malignant disease. The optimization principle (as low as reasonably achievable, ALARA) of article 56, when applied in a therapy situation, states that absorbed doses to nontarget tissues should be kept reasonably low, but not so low as to lose efficacy. We think that this applies above all to the fight against life-threatening cancer. As a consequence, we believe that to adhere to the optimization principle in oncological patients, nuclear medicine therapy should be based on individualized dosimetry.

\section{The maximum tolerable absorbed dose approach in nuclear medicine therapy}

In contrast to external beam radiotherapy, in nuclear medicine therapy we have patients or situations where the absorbed dose to 
the target volume cannot be calculated or reliably predicted for technical or practical reasons. For instance, metastatic lesions may not be measurable (being either too small or too infiltrative into normal tissue) or the molecule used in the tumour pretreatment dosimetry may have limited power to predict the absorbed dose of the therapeutic agent during treatment. In such circumstances there is no possibility for treatment optimization based on the absorbed dose to target tissues. The next best alternative is then to base therapy planning on the maximum tolerable absorbed dose (MTAD) to nontarget organs or tissues.

This is not a new concept in nuclear medicine therapy. In radioiodine therapy of metastatic thyroid cancer, the therapeutic activity was chosen to deliver the MTAD of 2 Gy to the red marrow. This approach was implemented more than 50 years ago by Benua et al. [1], and was successfully applied in the following decades by Leeper [2]. Unfortunately, the method has now been abandoned by many institutions, but it has continued in use in other centres [3-8]. The method provides solid indications of best outcome, in contrast to the fixed activity approach [3, 4], although a clinical study with the highest level of evidence (phase III randomized) has never been pursued. This approach was recently re-proposed [9], with the introduction of the acronym AHASA (as high as safely attainable) instead of ALARA. This type of approach thus aims to pursue the intended radiotherapeutic purpose of the exposure.

Another application of the AHASA approach is in ${ }^{131} \mathrm{I}-$ mIBG therapy of paediatric neuroblastoma, where the absorbed dose limit for acute myelodepression of 2 Gy (MTAD) to the red marrow was established by Fielding et al. in 1991 [10]. The VERITAS multicentre controlled trial is currently in development. It is based on individual patientbased maximization of the administered activity aiming to reach a total whole-body absorbed dose of $4 \mathrm{~Gy}$, in two tandem administrations 2 weeks apart [11]. Stem cell autologous transplantation is included in this study. A third example is the recently reported radioembolization of hepatocarcinoma with ${ }^{90} \mathrm{Y}$ glass microspheres [12], for which a treatment-planning paradigm was developed that aimed mainly to deliver the MTAD to the liver parenchyma, since the prediction of tumour absorbed dose can be uncertain [13].

The three examples discussed above (metastatic thyroid cancer, neuroblastoma, hepatocarcinoma) have the common strategy that, in order to pursue efficacy when the predictive power of tumour dosimetry is suboptimal, the maximization approach (AHASA) can be adopted instead of the optimization approach (ALARA). We think that in the presence of a potentially life-threatening disease, i.e. in oncology, when tumour absorbed dose cannot be reliably predicted, while there is the evident risk of radiation-induced toxicity to normal organs, therapeutic dose administrations according to the AHASA principle should be considered in clinical practice.

\section{Posology in current radiopharmaceutical registrations}

In the therapy of neuroendocrine tumours with radiolabelled somatostatin analogues, the radiopeptide DOTATATE labelled with ${ }^{177} \mathrm{Lu}$ is finally going to be registered. This is one of the most longed-for developments in nuclear medicine therapy in which radiopeptides have been used for 25 years, albeit as experimental agents. The observed clinical results indicate a net increase in overall survival compared with the nonlabelled somatostatin analogues (NETTER-1 study [14]). However, the administration schedule (posology) proposed in the registration study (7.4 GBq four times) may be a matter of concern if viewed from the perspective of optimization. Moving away from the "four cycles fits all" paradigm to an optimized prescription strategy would give many patients the opportunity to receive more treatment cycles and potentially obtain greater benefit from the treatment.

In the ongoing ILUMINET clinical study based on ${ }^{177}$ Lu-DOTATATE, the number of cycles is tailored to the absorbed dose to the kidneys in the individual patient, and in an interim analysis the number of delivered cycles was found to vary between three and eight [15]. In the group of patients in whom treatment was terminated because they had reached the protocol-specified dose limit, $73 \%$ received more and $9 \%$ received fewer than four cycles. In other words, it is possible that the results obtained in the registration study could be further developed. It is thus important that centres with dosimetric capability are not hindered from optimizing their treatment protocols and are allowed to tailor the number of cycles or the administered activity to the absorbed dose. The marked inter patient variations in peptide pharmacokinetics indicate the need of personalized treatment planning options [16-18].

It is clear that for most radiopharmaceuticals, the concept of posology is far from the optimization principle (ALARA), and even from the maximization principle (AHASA), and is therefore not "consistent with the intended radiotherapeutic purpose of the exposure". Even in patients in whom the intended purpose is purely palliative, there are indications that the patient benefit (response rate [3, 4] and overall survival [4, 19]) could be improved if the optimization (when possible) or maximization principles are adopted.

\section{Optimization principle in radioembolization procedures}

Liver radioembolization is a promising locoregional therapeutic option that has been approved using medical devices. It consists of the permanent implantation of radioactive microspheres under angiographic guidance. Because of the 
absence of biological clearance, dosimetry can be calculated with only one patient scan, in contrast to all other radiopharmaceuticals. As in external beam radiotherapy, simulation is possible using a nontherapeutic but radioactive biodegradable simulator $\left({ }^{99 \mathrm{~m}} \mathrm{Tc}\right.$ albumin macroaggregates). Radiationinduced toxicity to normal liver tissue is a considerable lifethreatening risk associated with this treatment $[20,21]$. Because dosimetry in liver radioembolization involves less work and this treatment is associated with an important risk, the number of centres in Europe currently adopting dosimetric planning of liver radioembolization is increasing (EANM survey 22]).

Garin et al. [23] make the following interesting comment about dosimetry in this field: "this type of powerful, pretherapeutic predictor of response and survival represents a clear advantage of radioembolization. This advantage is unfortunately not available with other therapeutic approaches used for liver cancer, such as chemotherapy, biotherapy, or chemoembolization". This "clear advantage", available in general with photon-emitting agents, is obviously not taken advantage of if a nondosimetry based posology is adopted. Tong et al. [24] make important additional remarks about nuclear medicine therapy in general: “... therapeutic radiopharmacenticals exert their biological response primarily by the deterministic effect of radiation, ... Any studies demonstrating apparent safety in spite of empirical or semi-empirical methods only leads to the logical conclusion that these methods have a conservative tendency to underdose, sacrificing efficacy for safety. ... The modern era of personalized medicine demands the measurement of patient-specific biophysical parameters to individualize treatment so as to maximize the desired effect while minimizing toxicity".

Interestingly, for the first time in the history of nuclear medicine therapy, all the companies supplying radiolabelled microspheres for liver radioembolization are making substantial efforts to optimize the treatment with dosimetry $[25,26]$ (https://www.btg-im.com/en-GB/Simplicit90Y/Home).

\section{The regulatory agency responsibility for the conflict between optimization and posology}

Nuclear medicine therapy is performed using radiopharmaceuticals or radioactive medical devices with the unique option of performing patient-specific treatment. The "pharmaceutical" or "drug" (medical device) properties of these agents are generally well acknowledged legally, and are carefully observed for the registration process. However, their radioactive nature is paradoxically neglected, despite this being their main mechanism of action [24]. The radiation mechanism clearly indicates that they belong to the field of radiotherapy (definition 81 in Directive 2013/59).
When regulatory agencies accept posology based only on fixed administrations, for instance as fixed activities and a fixed number of cycles $\left({ }^{177} \mathrm{Lu}\right.$ DOTATATE), or a fixed activity per body mass $\left({ }^{223} \mathrm{Ra}\right)$, in order to comply with the package insert, therapists may be prevented from basing their prescriptions on the individual patient absorbed dose. As a consequence, the application of the optimization principle (article 56) of radiotherapy is prevented. In other words, package inserts containing only nondosimetric posology force therapists into conflict with article 56.

We consider that the responsibility for this lies with the registration and health authorities, who should be well aware of the above arguments before licensing a nondosimetric posology, excluding the possibility of dosimetry-based optimization.

In the present situation, where internal dosimetry is still not fully applied, we propose that the package insert of any radioactive agent for therapy should indicate, in parallel with conventional posology, a dosimetry-based administration undertaken under the full responsibility of the therapy team. Otherwise the optimization requirement will not be met for nuclear medicine therapy. A complete quality assurance programme should be in place to ensure accurate delivery of any prescribed treatment.

\section{Compliance with ethical standards}

Funding This study was not funded.

Ethical approval This article does not describe any studies with human participants or animals performed by any of the authors.

Conflict of interest C. Chiesa has received speaker and consultant honoraria, and sponsorship to congresses from BTG Biocompatibles. He has received a consultant honorarium from Medpace CRO.

G. Flux received research grants from Sirtex and from BTG Biocompatibles.

S. Walrand has two research projects funded by Sirtex.

$\mathrm{M}$. Bardiès has received a speaker honorarium from BTG Biocompatibles and is a consultant for Roche.

M. Lassmann has received research grants from Ipsen, Nordic Nanovector and Siemens.

$\mathrm{K}$. Bacher has received a one-day consultant honorarium from BTG Biocompatibles that he transferred to Ghent University.

E.P. Visser has received research grants from Siemens.

K. Sjögreen Gleisner, L. Strigari, U. Eberlein, N. Couin, J. Gear, M. Ljungberg, and M. Konijnenberg declare that they have no conflicts of interest.

\section{References}

1. Benua RS, Cicale NR, Sonenberg M, Rawson RW. The relation of radioiodine dosimetry to results and complications in the treatment of metastatic thyroid cancer. Am J Roentgenol Radium Ther Nucl Med. 1962;87:171-82. 
2. Leeper RD. The effect of 131 I therapy on survival of patients with metastatic papillary or follicular thyroid carcinoma. J Clin Endocrinol Metab. 1973;36:1143-52.

3. Lee JJ, Chung JK, Kim SE, Kang WJ, Park DJ, Lee DS, et al. Maximal safe dose of 131I after failure of standard fixed dose therapy in patients with differentiated thyroid carcinoma. Ann Nucl Med. 2008;22:727-34.

4. Klubo-Gwiedzinska J, Van Nostrand D, Atkins F, Burman K, Jonklaas J, Mete M, et al. Efficacy of dosimetric versus empiric prescribed activity of 131I for therapy of differentiated thyroid cancer. J Clin Endocrinol Metab. 2011;96:3217-25.

5. Verburg FA, Hänscheid H, Biko J, Hategan MC, Lassmann M, Kreissl MC, et al. Dosimetry-guided high-activity 131I therapy in patients with advanced differentiated thyroid carcinoma: initial experience. Eur J Nucl Med Mol Imaging. 2010;37:896-903.

6. Bianchi L, Baroli A, Lomuscio G, Pedrazzini L, Pepe A, Pozzi L, et al. Dosimetry in the therapy of metastatic differentiated thyroid cancer administering high 131I activity: the experience of Busto Arsizio hospital (Italy). Q J Nucl Med Mol Imaging. 2012;56: 515-21.

7. Dorn R, Kopp J, Vogt H, Heidenreich P, Carrol RG, Gulec SA. Dosimetry-guided radioactive iodine treatment in patients with metastatic differentiated thyroid cancer: largest safe dose using a riskadapted approach. J Nucl Med. 2003;44:451-6.

8. Hartung-Knemeyer V, Nagarajah J, Jentzen W, Ruhlmann M, Freudenberg LS, Stahl AR, et al. Pre-therapeutic blood dosimetry in patients with differentiated thyroid carcinoma using 124-iodine: predicted blood doses correlate with changes in blood cell counts after radioiodine therapy and depend on modes of TSH stimulation and number of preceding radioiodine therapies. Ann Nucl Med. 2012;26(9):723-9.

9. Verburg FA, Luster M, Giovanella L, Lassmann M, Chiesa C, Chouin N, et al. The "reset button" revisited: why high activity 131I therapy of advanced differentiated thyroid cancer after dosimetry is advantageous for patients. Eur J Nucl Med Mol Imaging. 2017;44(6):915-7.

10. Fielding SL, Flower MA, Ackery D, Kemshead JT, Lashford LS, Lewis I. Dosimetry of iodine 131 metaiodobenzylguanidine for treatment of resistant neuroblastoma: results of a UK study. Eur J Nucl Med. 1991;18:308-16.

11. Buckley SE, Saran FH, Gaze MN, Chittenden S, Partridge M, Lancaster D, et al. Dosimetry for fractionated 131I-mIBG therapies in patients with primary resistant high-risk neuroblastoma: preliminary results. Cancer Biother Radiopharm. 2007;22(1):105-12.

12. Chiesa C, Mira M, Maccauro M, Spreafico C, Romito R, Morosi C, et al. Radioembolization of hepatocarcinoma with $90-\mathrm{Y}$ glass microspheres: development of an individualized treatment planning strategy based on dosimetry and radiobiology. Eur J Nucl Med Mol Im. 2015;42:1718-38.

13. Gnesin S, Canetti L, Adib S, Cherbuin N, Silva Monteiro M, Bize P, et al. Partition model-based 99mTc-MAA SPECT/CT predictive dosimetry compared with $90 \mathrm{Y}$ TOF PET/CT posttreatment dosimetry in radioembolization of hepatocellular carcinoma: a quantitative agreement comparison. J Nucl Med. 2016;57(11):1672-8.

14. Strosberg J, El-Haddad G, Wolin E, Hendifar A, Yao J, Chasen B, et al. Phase 3 trial of $177 \mathrm{Lu}$-Dotatate for midgut neuroendocrine tumors. N Engl J Med. 2017;376(2):125-35.

15. Sundlöv A, Sjögreen-Gleisner K, Svensson J, Ljungberg M, Olsson $\mathrm{T}$, Bernhardt $\mathrm{P}$, et al. Individualised 177Lu-DOTATATE treatment of neuroendocrine tumours based on kidney dosimetry. Eur J Nucl Med Mol Imaging. 2017. doi:10.1007/s00259-017-3678-4.

16. Barone R, Borson-Chazot F, Valkema R, Walrand S, Chauvin F, Gogou L, et al. Patient-specific dosimetry in predicting renal toxicity with 90Y-DOTATOC: relevance of kidney volume and dose rate in finding a dose-effect relationship. J Nucl Med. 2005;46:99S$106 \mathrm{~S}$.

17. Kletting P, Kull T, Maaß C, Malik N, Luster M, Beer AJ, et al. Optimized peptide amount and activity for 90Y-labeled DOTATATE therapy. J Nucl Med. 2016;57:503-8.

18. Bergsma H, Konijnenberg MW, van der Zwan WA, Kam BL, Teunissen JJ, Kooij PP, et al. Nephrotoxicity after PRRT with (177)Lu-DOTA-octreotate. Eur J Nucl Med Mol Imaging. 2016;43(10):1802-11.

19. Chiesa $\mathrm{C}$. The individualized dosimetry in the radioembolization of hepatocarcinoma with 90Y-microspheres [abstract]. Phys Med. 2016;32 Suppl 3:169-70. 10.1016/j.ejmp.2016.07.264

20. Cremonesi M, Chiesa C, Strigari L, Ferrari M, Botta F, Guerriero F, et al. Radioembolization of hepatic lesions from a radiobiology and dosimetric perspective. Front Oncol. 2014;4:210

21. Mazzaferro V, Sposito C, Bhoori S, Romito R, Chiesa C, Morosi C, et al. Yttrium90 radioembolization for intermediate-advanced hepatocarcinoma: a phase II study. Hepatology. 2013;57(5):182637.

22. Sjogreen Gleisner K. Current status and preparedness to comply to the Directive (report on European survey). Eur J Nucl Med Mol Imaging. 2016;43(Suppl 1):S22 OP052.

23. Garin E, Boucher E, Rolland Y. 99mTc-MAA-based dosimetry for liver cancer treated using 90Y-loaded microspheres: known proof of effectiveness. J Nucl Med. 2014;55:1391-2.

24. Tong AK, Kao YH, Too CW, Chin KF, Ng DC, Chow PK. Yttrium90 hepatic radioembolization: clinical review and current techniques in interventional radiology and personalized dosimetry. $\mathrm{Br}$ J Radiol. 2016;89:20150943.

25. Willowson KP, Tapner M, The QUEST Investigator Team, Bailey DL. A multicentre comparison of quantitative 90Y PET/CT for dosimetric purposes after radioembolization with resin microspheres: the QUEST Phantom Study. Eur J Nucl Med Mol Imaging. 2015;42(8):1202-22.

26. Smits ML, Nijsen JF, van den Bosch MA, Lam MG, Vente MA, Mali WP, et al. Holmium-166 radioembolisation in patients with unresectable, chemorefractory liver metastases (HEPAR trial): a phase 1, dose-escalation study. Lancet Oncol. 2012;13:1025-34. 